\title{
Stellar populations in star forming galaxies in the Sloan Digital Sky Survey
}

\author{
Pieter Westera $^{1,2}$, François Cuisinier ${ }^{3}$ and Carlos R. Rabaça ${ }^{3}$ \\ ${ }^{1}$ Observatório Nacional, Rio de Janeiro, Brazil \\ email: pieter@on.br \\ ${ }^{2}$ Universidade Estadual de Santa Cruz, Ilhéus, Bahia, Brazil \\ ${ }^{3}$ Observatório do Valongo, Universidade Federal do Rio de Janeiro, Brazil \\ email: francois@astro.ufrj.br, rabaca@astro.ufrj.br
}

\begin{abstract}
We examine the star forming phenomenon as it can be encountered in galaxies in the Sloan Digital Sky Survey, which possibly contains the largest homogeneous sample of star forming galaxy spectra to date.

After eliminating all spectra with an insufficient signal-to-noise ratio, without strong emission lines, and without the [OII $\lambda 3727 \AA$ line, which is necessary for the determination of the gas metallicity (which excludes galaxies with redshift $\lesssim 0.024-0.025$ ), our sample contains $\sim 6000$ spectra of star forming galaxies.

Through a detailed stellar population analysis employing evolutionary synthesis methods we determined the stellar composition of these galaxies, that is, the masses, ages and metallicities of their partial stellar populations.

We find that most, possibly all, galaxies of our sample contain, apart from the presently bursting, ionising young generation $\left(\leqslant 10^{7} \mathrm{yrs}\right)$, old $\left(\geqslant 10^{9} \mathrm{yrs}\right)$ and intermediate (between $10^{7}$ and $10^{9} \mathrm{yrs}$ ) populations, whereas the old population dominates the stellar mass (but not the light).

We also find that high (stellar) mass galaxies have higher gas metallicities and lower present star formation rates relative to their total (stellar) masses, than low mass galaxies, indicating a higher chemical evolution degree for high mass galaxies.

Furthermore, we find that gas enrichment mechanisms in star forming galaxies do not vary with galactic mass, being the same for low- and high-mass galaxies on average. Gas enrichment mechanisms seem to present a greater variety at the high-mass end, though, indicating a more complex assembly history for high-mass galaxies.
\end{abstract}

Keywords. galaxies: starburst, galaxies: stellar content, ISM: abundances, galaxies: abundances, galaxies: formation, galaxies: evolution

\section{Introduction}

Star forming galaxies are galaxies in a star formation phase, which results in strong emission lines in their spectra, particularly from hydrogen and helium, but also from heavier elements (Ne, N, O, Ar, S, etc). However, their spectra also show a continuum contribution, fainter than the emission lines, but clearly visible, which is attributed to stars.

In this work, we analyse by means of a population synthesis the stellar composition of a sample of over 6000 star forming galaxy spectra from the Sloan Digital Sky Survey (SDSS). The selected spectra have high signal-to-noise ratios and strong oxygen emission lines, permitting the determination of the gas metallicity using the $\mathrm{P}$ method by Pilyugin (2001). 


\section{Method}

We separated the galaxies of our sample from galaxies with active galactic neuclei (AGNs) using the line excitation criterion by Kewley et al. (2001).

We determined the stellar compositions of these galaxies using evolutionary population synthesis - and spectral fitting methods, already used in Cuisinier et al. (2006) and Lisker et al. (2006), decomposing the spectra into three single stellar populations (SSPs), a young one (aged less than $10 \mathrm{Myr}$ ), one of intermediate age (between $10 \mathrm{Myr}$ and 1 Gyr), and an old one (older than 1 Gyr), of which we determined the (relative) masses, ages, and metallicities.

\section{Results}

We find that most star forming galaxies, if not all, of our sample contain, apart from the presently forming young ( $<10 \mathrm{Myr}$ ) bright population, old ( $>1 \mathrm{Gyr}$ ) - and intermediateage (between $10 \mathrm{Myr}$ and $1 \mathrm{Gyr}$ ) populations, with the old population in general constituting over $90 \%$ of the stellar mass, whereas the young population makes up for less than $1 \%$ of the stellar mass.

The average metallicity of the gas and of the young - and intermediate populations is around -0.4 , while the one of the old population lies close to -0.7 .

We also find that, at present, high mass galaxies have higher (gas) metallicities and lower fractional star formation rates (SFRs) than low mass galaxies, in agreement with the "downsizing" scenario.

The fact, that the metal enrichment since the formation of the old population, $[\mathrm{Fe} / \mathrm{H}]_{y / i}-$ $[\mathrm{Fe} / \mathrm{H}]_{o}$, does not depend on the galaxy mass, suggests, that the enrichment mechanism are the same for low- and high-mass galaxies.

However the dispersion of $[\mathrm{Fe} / \mathrm{H}]_{y / i}-[\mathrm{Fe} / \mathrm{H}]_{o}$ does increase with galactic mass, indicating more complex chemical (and certainly assembly) histories for high mass galaxies.

We calculated the "global" SFR, that is, summed up over all galaxies of our sample. Comparisons with simulations of galaxies with different star fromation histories show, that this "global" SFR is consistent with the a constant SFR. We conclude that in these galaxies, and in galaxies in general, star formation happens on an approximately constant level on long timescales, in spite of its bursty nature on short timescales.

\section{Conclusions}

This leads to the conclusion that star forming galaxies are, in fact, old objects caught in a star formation phase, and that their star formation, and the one of galaxies in general, in spite of occurring in bursts, happens on an approximately constant level on longer timescales. We found indications that the gas recycling mechanisms are similar in low - and in high mass galaxies, as well as in galaxies with low - and high present-day metallicities, whereas the chemical - and assembly history might be more complicated in high mass galaxies. We also confirm a "downsizing" scenario for our galaxy sample.

\section{References}

Cuisinier, F., Westera, P., Telles, E., \& Buser, R. 2006, A\& A, 455, 825

Kewley, L. J., Dopita, M. A., Sutherland, R. S., Heisler, C. A., \& Trevena, J. 2001, ApJ, 556, 121

Lisker, T., Glatt, K., Westera, P., \& Grebel, E. K. 2006, AJ, 132, 2432

Pilyugin, L. S. 2001, A\& $A$, 369, 594 